\title{
Remembering things past
}

\author{
Memory is the signature property of the adaptive response, and vaccination is a hugely important medical intervention- \\ understanding the former will help perfect the latter.
}

$\mathrm{T}$ he ability to remember a specific pathogen and to respond more rapidly after subsequent exposure to it is the defining feature of adaptive immunity. In lay terms, at least, the idea of immunity has become synonymous with memory: an animal is said to be 'immune' if they have a strong memory response. Vaccination depends fundamentally on immunological memory, and the potency of certain vaccines attests to the pre-eminence of memory as the cornerstone of the vertebrate immune response. Indeed, vaccination is one of the most important medical interventions ever developed and has arguably prevented more deaths than any other in human history. The successful eradication of smallpox and possible near-term eradication of polio by effective vaccination programs stand as fitting testaments to the potential of this approach. However, since the time of Jenner and Pasteur, the development of vaccines has been a rather hit-or-miss affair with little or no understanding of the mechanisms that underpin effective vaccination. Light is now being shone into this black box by insights in adjuvant formulation, innate immunity and antigen presentation. Moreover, it is becoming increasingly apparent that the next generation of vaccines will depend crucially on fuller understanding of the mechanisms and processes that give rise to immunological memory.

The importance of immunological memory for vaccinology and the rapid progress in this area have inspired the present focus issue (http:// www.nature.com/ni/focus/immunologicalmemory/). The eight pieces in this immunological focus cover $\mathrm{T}$ cell and $\mathrm{B}$ cell memory, immune exhaustion, factors involved in the generation of memory, the physical compartmentalization of memory, the development of new vaccines and the topic of natural killer (NK) cell memory. A thread connecting all these reviews, succinctly summed up by Michael Bevan in his overview, is that understanding memory enables the design of better vaccines.

The cellular and molecular events that lead to the generation $\mathrm{CD} 8^{+}$ $\mathrm{T}$ cell memory are relatively well defined; however, the same cannot be said about $\mathrm{CD} 4^{+}$helper $\mathrm{T}$ cells. This has led some to suggest that memory $\mathrm{CD} 4^{+}$helper T cells may not even exist-at least, not as a specialized long-lived population. Marion Pepper and Marc Jenkins, however, outline strong evidence that differentiated cells such as those of the $T_{H} 1, T_{H} 2$ and $T_{H} 17$ subsets of helper $T$ cells can give rise to stable populations of effector memory $\mathrm{T}$ cells. These cells express homing receptors that allow migration to sites of inflammation and also rapidly produce a variety of effector cytokines such as interferon- $\gamma$ and interleukin 4 . Central memory cells $\mathrm{T}$ cells do not produce any of the prototypic effector cytokines (at least early in the response) but can instead expand their populations rapidly and express receptors for chemokines and adhesion molecules that facilitate recirculation through secondary lymphoid organs. How a cell 'chooses' to become a central memory or effector memory $\mathrm{T}$ cell is hotly debated, and Pepper and Jenkins argue for the involvement of $\mathrm{B}$ cells and follicular helper $\mathrm{T}$ cells in this process. Indeed, interactions between B cells and follicular helper $\mathrm{T}$ cells are key to the generation of humoral memory responses and are the subject of the review by Stephen Nutt and David Tarlinton.

How memory cells are maintained in the long term is another crucial question. Jonathan Sprent and Charles Surh argue that common $\gamma$-chain cytokines such as interleukin 7 are responsible for not only the homeostatic maintenance of memory cells but also their generation. These cytokines, in conjunction with low-intensity triggering of the $T$ cell antigen receptor (such as by self antigen), lead to the generation of cells with a memory phenotype. This process gives rise to the surprising finding that memory cells may exist even before exposure to pathogen.

Immunologists have become increasingly aware of the fact that immune responses are compartmentalized; that is, distinct effector populations are generated by the local tissue microenvironment. Brian Sheridan and Leo Lefrançois discuss the signals responsible for generating these localized responses and how memory cells residing in nonlymphoid tissues such as the skin and mucosa can act as the first line of defense against microbial infection. Adaptive immune responses need to be tightly controlled, but failure to achieve this can result in chronic $\mathrm{T}$ cell stimulation, which gives rise to so-called 'exhausted T cells' John Wherry argues that these poorly functional cells represent a phenotypically distinct state and can be a prominent feature of both chronic infection and cancer. Exhausted cells are also associated with aging, and their presence is probably an important factor to consider when vaccinating the elderly.

Memory is traditionally thought to be a feature unique to $B$ cells and T cells; however, Silke Paust and Ulrich von Andrian discuss the evidence of memory in the third main type of lymphocyte: NK cells. Data suggest that memory-like properties can be ascribed to NK cells in experimental delayed-type hypersensitivity responses and certain viral infections, and it seems that these cells reside mainly in the liver. Although on one level the proposal of memory NK cells may seem controversial, it is important to emphasize that memory-like phenomena can be observed even in certain invertebrates not normally considered to have adaptive immune responses. Whether memory NK cells represent a true counterpart to $\mathrm{B}$ cell and $\mathrm{T}$ cell memory and whether or not they have an important role in physiological immune responses remains to be determined.

Finally, Bali Pulendran and Rafi Ahmed discuss how such fundamental insights into immunological memory can be translated into the production of vaccines. How can the most appropriate immune response be elicited by vaccination, whether cell mediated or humoral, and in which compartment-systemically or at specific tissue sites such as the gut mucosa? What is the best ' readout' for determining vaccine efficacy? Perhaps most importantly, how can robust and long-lived responses be generated? New insights into immunological memory will inform the better design of vaccines and could lead to the prevention of previously intractable diseases. 